\title{
Clinical validation of a non-heteronormative version of the Social Interaction Anxiety Scale (SIAS)
}

\author{
Philip Lindner ${ }^{1 *}$, Christopher Martell ${ }^{2}$, Jan Bergström ${ }^{3}$, Gerhard Andersson ${ }^{1,4}$ and Per Carlbring ${ }^{3}$
}

\begin{abstract}
Introduction: Despite welcomed changes in societal attitudes and practices towards sexual minorities, instances of heteronormativity can still be found within healthcare and research. The Social Interaction Anxiety Scale (SIAS) is a valid and reliable self-rating scale of social anxiety, which includes one item (number 14) with an explicit heteronormative assumption about the respondent's sexual orientation. This heteronormative phrasing may confuse, insult or alienate sexual minority respondents. A clinically validated version of the SIAS featuring a non-heteronormative phrasing of item 14 is thus needed.
\end{abstract}

Methods: 129 participants with diagnosed social anxiety disorder, enrolled in an Internet-based intervention trial, were randomly assigned to responding to the SIAS featuring either the original or a novel non-heteronormative phrasing of item 14, and then answered the other item version. Within-subject, correlation between item versions was calculated and the two scores were statistically compared. The two items' correlations with the other SIAS items and other psychiatric rating scales were also statistically compared.

Results: Item versions were highly correlated and scores did not differ statistically. The two items' correlations with other measures did not differ statistically either.

Conclusions: The SIAS can be revised with a non-heteronormative formulation of item 14 with psychometric equivalence on item and scale level. Implications for other psychiatric instruments with heteronormative phrasings are discussed.

Keywords: Heteronormativity, Social Interaction Anxiety Scale, Psychometric

\section{Introduction}

Heteronormativity refers to practices that both originate in and reproduce presumptions and norms of there being two distinct, opposite sexes-men and women-and that these two sexes are attracted to each other (i.e. heterosexuality) without considering other sex-attraction constellations, e.g. same-sex or attraction independent of sex [1]. Despite recent changes in Western societal attitudes and practices towards non-heterosexual orientations and the individuals identified therewith (referred to as sexual minorities) [2], research suggests that sexual minorities may still experience what they consider to be inappropriate heteronormative responses from the medical-clinical community [3,4]. Heteronormative behaviours may perplex sexual minority patients resulting in

\footnotetext{
* Correspondence: philip.lindner@ki.se

${ }^{1}$ Department of Clinical Neuroscience, Karolinska Institutet, Karolinska, Sweden

Full list of author information is available at the end of the article
}

communication problems, or worse, offend or embarrass sexual minority patients, alienating them from healthcare services [4-6]. Identifying heteronormative practices and replacing them with inclusive ones should therefore be made a priority for all healthcare based upon patient equality.

The increased risk of being ostracised and harassed by others has been proposed as an explanation for the finding that sexual minority individuals are at greater risk for developing psychiatric disorders [7-10]. Specifically related to their psychosocial situation, sexual minority individuals appear to show increased levels of social anxiety $[6,11]$, i.e. a fear of negative evaluations and rejection in social situations [12]. These findings stress the importance of having screening instruments for social anxiety, validated for use with sexual minority individuals in a clinical setting.

\section{Biomed Central}


A commonly used self-rating scale in screening for social anxiety is the Social Interaction Anxiety Scale (SIAS), developed by Mattick and Clarke [13]. The SIAS assesses fears associated with social interactions, such as sounding stupid and being ignored [13]. Item 14 of the SIAS aims to capture the anxiety associated with communicating with an attractive individual who-on the basis of sex-could be a potential partner. Unfortunately, the current phrasing of this item assumes a heterosexual orientation of the respondent: "I have difficulty talking to attractive persons of the opposite sex" [13]. For individuals who do not equate the trait of attractiveness with the opposite sex, the current phrasing creates a forced arbitrary choice of rating the difficulty of talking to either attractive people, or people of the opposite sex. This ambiguity of what is measured may lower the validity of the item and scale when used by sexual minority respondents. The current heteronormative phrasing may also insult, embarrass or demoralise sexual minority respondents, constituting an ethical problem in administering the instrument. Indirect evidence suggests that simply omitting the words "of the opposite sex" (as done in one study [11]) may yield a different interpretation of the item. While male and female ratings of male and female attractiveness tend to correlate well, heterosexual ratings of the opposite sex are more likely to include a judgment on the desirability as a potential romantic and/or sexual partner [14]. Accordingly, the original heteronormative phrasing of item 14 of the SIAS likely reflects an attempt to capture the difficulty of talking to an attractive potential partner, and not merely someone attractive. A novel, non-heteronormative phrasing of item 14 should reflect this important aspect.

Of note, none of the shortened versions of the SIAS that have been independently developed through item and factor analysis of the original instrument, have included the original item 14 [15-18]. However, there are several justifications for the continued use of the original version. The full SIAS remains popular in both clinical practice and research and using the full SIAS ensures comparability with previous findings and practices (e.g. clinical cut-offs). Further, the screening accuracies of these novel instrument versions have yet to be adequately researched, or compared. When used in research settings, were a greater span of possible results enables a greater power to detect significant differences, the original 20-item SIAS version should likewise be preferred. Hence, there are compelling reasons to continue using the full SIAS version, which requires revising the problematic 14th item with a non-heteronormative phrasing.

The purpose of this psychometric study was to develop and validate such a phrasing in a clinical sample. Our hypothesis was that our novel, non-heteronormative phrasing would yield psychometric properties similar to the original phrasing.

\section{Material and methods \\ Participants}

Participants were 129 individuals enrolled in a larger, two-phase randomised controlled trial featuring an Internet-administered attention bias modification program $(\mathrm{ABM})$ and therapist-guided cognitive behavioural self-help for social anxiety disorder [19,20]. Demographic and clinical characteristics are presented in Additional file 1: Table S1 in the Supplementary material. All participants included in the intervention study fulfilled diagnostic criteria for social anxiety disorder (SAD) at initial screening, as assessed by a Structured Clinical Interview for DSM-IV axis I disorder (SCID; [21]), conducted by telephone [22]. The intervention study was approved by the Swedish Ethical Review Board (2012-132-31Ö) and preregistered in the Clinicaltrials.gov registry (NCT01570400). See the published study protocol [19] or the primary results of the trial [20] for more details.

\section{Procedure}

The data collection for this study was done after the two initial weeks of ABM, before the nine-week, therapistassisted cognitive behavioural self-help program commenced. Of note, unlike in the primary analysis of the trial [20] which excluded participants who had commenced parallel treatments $(n=120)$, we used all available data from this measurement point $(n=129)$. The group assignments used in the primary analysis of the trial were not used in the present study.

After their last session of ABM, the participants were asked to once again fill out the same rating scales (including the SIAS) as was done at screening, after which the cognitive behavioural therapy would commence. Before answering the SIAS questions, participants were asked whether their birthday was on an odd or even day. This divided participants into two groups: one group that responded to the SIAS with the original item 14 ("the Original item 14 first-group"), and one group that responded to the SIAS with the novel item 14 ("the $\mathrm{Re}$ vised item 14 first-group"). This method of randomisation was chosen because it was (a) technically feasible; (b) since it should in theory create a half-half division; and (c) because there is no reason-theoretical or empirical-to suspect that the parity of one's birthday has any influence whatsoever on the factors relevant for this study. Having responded to the complete SIAS version to which they were randomised, all respondents were then asked the other version of item 14. Thus, all participants responded to both versions of item 14, allowing within-subject analyses. Splitting the sample into two groups was done to enable investigating effects of 
administration order (methods and results described in the Supplementary material). All calculations described in the main manuscript were performed on the sample undivided.

\section{Instruments}

All measurements were made using a dedicated, secure online interface. Previous psychometric research has validated the Internet-administration of the measures used in this study [23-26] with the presentation format of one item per page [27]. All items were mandatory to ensure no missing data.

The Social Interaction Anxiety Scale (SIAS; [13]) uses 20 items and a 5-point Likert scale (scored 0-4) with verbal descriptors to assess to what degree the respondent agrees with a statement related to interaction-related characteristics of social anxiety. The SIAS has demonstrated good internal consistency, test-retest reliability, convergent validity and diagnostic accuracy $[13,28,29]$. The revised, non-heteronormative phrasing of SIAS item 14 was developed by authors PL and PC to capture the difficulty of talking to an attractive potential partner, without a heteronormative assumption regarding the sexual orientation of the respondent. The final wording settled upon was: "I have difficulty talking to attractive persons of the sex/sexes that I am interested in" (translation from Swedish). The phrasing allows for respondents of all sex-attraction constellations to answer the question without excluding the potential partner connotation of the original version yet remaining semantically similar to the original phrasing ("I have difficulty talking to attractive persons of the opposite sex"; see Discussion below). Comparable readability for the two item versions was assessed using the Flesch Reading Ease and FleschKincaid Grade Level tests (as implemented in Microsoft Word 2010). These scores were similar: reading ease score for the revised phrasing was 47.8 , compared to a score of 41.8 for the original phrasing (with higher scores indicating easier reading), while grade level score for the revised phrasing was 10.5, compared to a score of 10.1 for the original phrasing.

Other instruments administered at the same occasion provided additional measures of mental health and quality of life: the self-rated Liebowitz Social Anxiety Scale (LSAS-SR; [30,31]), the Social Phobia Scale (SPS; [13]), the self-rated Montgomery-Åsberg Depression Rating Scale (MADRS-S; [32]) and the Quality of Life Inventory [33-35]. Participants also provided free-text answers to a question on the importance of the other person's sex on experienced social anxiety (see Additional file 1). This was done to confirm the importance of keeping the potential partner connotation in our revised phrasing of item 14.

\section{Calculations and analyses}

Statistical analyses were performed using IBM SPSS version 22 and the $R$ statistical environment (http:// www.r-project.org), running on Windows 7. Following the recommendations outlined by Carifio and Perla [36], scale-level data was treated as interval-level data (allowing parametric tests) while individual items were not.

Psychometric equivalence of the two item versions was assessed using direct statistical comparison with the Wilcoxon Signed Ranks test and calculating the Spearman's rank correlation between the two item versions. We also calculated the correlations between each item version and all other SIAS items to assess whether the internal consistency would differ. Both item versions were also correlated with the item-corrected total SIAS score (excluding any version of item 14 to avoid statistical dependency) and the other self-rating scales to ensure similar convergent validities. The two items' correlation coefficients were then statistically compared using Steiger's Z test [37], as implemented in the cocor R package (http://cran.r-project.org/package=cocor) [38].

Additionally, item- and scale-level data for the two groups were compared to investigate possible effects of administration order. Further details and results are described in the Supplementary material. Also included in the Supplementary material are methods and results pertaining to the analyses of free-text answers regarding the impact of sex on social anxiety. Scale-level data (total scores and internal consistencies) for the SIAS (either with the original $\left(w / o_{14}\right)$ or revised $\left(w / r_{14}\right)$ item 14 included) and the other scales are also included in the Supplementary material, both for the sample undivided and group-wise.

\section{Results}

\section{Equivalence of items}

The median difference in scores between the two item 14 versions was 0 , with the large majority of respondents $(\mathrm{n}=99)$ giving the same answer to both item versions (i.e. no difference in scores). The Wilcoxon Signed Ranks test was convincingly insignificant $(p=0.87)$. The correlation between item versions was $r_{s}=0.912$. Steiger's $Z$ test revealed no differences in correlations to other measures between the two item 14 versions (all $p>0.117$ ). See Table 1 for details.

\section{Additional analyses}

The two administration-order groups did not differ in any item 14 scores, suggesting no effect of administration order. A majority of respondents $(n=84)$ answered "Yes" to the question "Do you find it more difficult talking to an attractive person if this person, on the basis of sex, is a potential partner?" and these respondents reported higher values on both item 14 versions. Viz., potential partner 
Table 1 Comparison of correlation coefficients

\begin{tabular}{|c|c|c|c|c|}
\hline \multirow[t]{2}{*}{ Measure } & \multirow[t]{2}{*}{$\begin{array}{l}\text { Correlation } \\
\text { with original } \\
\text { item } 14\end{array}$} & \multirow[t]{2}{*}{$\begin{array}{l}\text { Correlation } \\
\text { with revised } \\
\text { item } 14\end{array}$} & \multicolumn{2}{|c|}{$\begin{array}{l}\text { Statistical } \\
\text { comparison of } \\
\text { correlation } \\
\text { coefficients }\end{array}$} \\
\hline & & & $z$ & $p$ \\
\hline Original SIAS item 14 & & $.912^{* *}$ & & \\
\hline Revised SIAS item 14 & $.912^{* *}$ & & & \\
\hline Item-corrected SIAS & $.497^{* *}$ & $.479^{* *}$ & 0.556 & 0.578 \\
\hline SIAS item 1 & $.424^{* *}$ & $.394^{* *}$ & 0.884 & 0.377 \\
\hline SIAS item 2 & $.487^{* *}$ & $.454^{* *}$ & 1.007 & 0.314 \\
\hline SIAS item 3 & $.236^{* *}$ & $.241^{* *}$ & -0.138 & 0.890 \\
\hline SIAS item 4 & $.404^{* *}$ & $.413^{* *}$ & -0.265 & 0.791 \\
\hline SIAS item 5 & $.238^{* *}$ & $.210^{*}$ & 0.770 & 0.442 \\
\hline SIAS item 6 & $.442^{* *}$ & $.389^{* *}$ & 1.567 & 0.117 \\
\hline SIAS item 7 & $.316^{* *}$ & $.298^{* *}$ & 0.507 & 0.612 \\
\hline SIAS item 8 & $.449^{* *}$ & $.414^{* *}$ & 1.044 & 0.297 \\
\hline SIAS item 9 & $.231^{* *}$ & $.215^{*}$ & 0.440 & 0.660 \\
\hline SIAS item 10 & $.399^{* *}$ & $.379^{* *}$ & 0.583 & 0.560 \\
\hline SIAS item 11 & $.312^{* *}$ & $.296^{* *}$ & 0.450 & 0.652 \\
\hline SIAS item 12 & $.264^{* *}$ & $.259^{* *}$ & 0.139 & 0.890 \\
\hline SIAS item 13 & $.207^{*}$ & $.252^{* *}$ & -1.239 & 0.215 \\
\hline SIAS item 15 & $.376^{* *}$ & $.357^{* *}$ & 0.548 & 0.584 \\
\hline SIAS item 16 & $.478^{* *}$ & $.427^{* *}$ & 1.540 & 0.124 \\
\hline SIAS item 17 & $.256^{* *}$ & $.285^{* *}$ & -0.808 & 0.419 \\
\hline SIAS item 18 & .127 & .145 & -0.486 & 0.627 \\
\hline SIAS item 19 & $.329^{* *}$ & $.359^{* *}$ & -0.858 & 0.391 \\
\hline SIAS item 20 & $.302^{* *}$ & $.296^{* *}$ & 0.169 & 0.866 \\
\hline LSAS-SR & $.446^{* *}$ & $.426^{* *}$ & 0.598 & 0.550 \\
\hline SPS & $.302^{* *}$ & $.252^{* *}$ & 1.395 & 0.163 \\
\hline MADRS-S & $.388^{* *}$ & $.344^{* *}$ & 1.270 & 0.204 \\
\hline QOLI & $-.296^{* *}$ & $-.287^{* *}$ & -0.252 & 0.801 \\
\hline
\end{tabular}

${ }^{*} p<0.05,{ }^{* *} p<0.01$. Acronyms: SIAS, Social Interaction Anxiety Scale; LSAS-SR, Liebowitz Social Anxiety Scale self-rated; SPS, Social Phobia Scale; MADRS-S, Montgomery-Åsberg Depression Rating Scale self-rated; QOLI, Quality of Life Inventory.

connotation appears to influence self-perceived social anxiety, at least for some. See the Additional file 1 for detailed results.

\section{Discussion}

Heteronormativity in psychiatric and psychological practice is an under-discussed topic that is receiving increasing attention. The present study was undertaken to examine whether it was possible to revise the phrasing of item 14 of the Social Interaction Anxiety Scale (SIAS) into a non-heteronormative one that would not insult, alienate or confuse sexual minority respondents, with retained psychometric properties. Nothing in our results suggests that our novel SIAS item 14 performed differently to the original item version. We therefore conclude that the SIAS can be revised with our non-heteronormative version of item 14 without loss of psychometric compatibility with previous research and clinical practice (e.g. clinical cut-offs) derived from use of the original SIAS.

Our findings are in line with those of Weiss et al. [39] who explored four alternative rephrased versions of the same SIAS item 14. The phrasing "I have difficulty talking to a potential romantic partner" was chosen as the preferred revised version due to the near-exact equivalence of scores. Out of the three other options explored-“... an attractive person", "... someone I could date" and "... someone I'm attracted to"-only the latter differed significantly $(p<0.001)$ in scores to the original item. Semantically, our novel phrasing, "I have difficulty talking to attractive persons of the sex/sexes that I am interested in", can be considered to fall in-between of Weiss et al.'s preferred and discouraged alternatives, incorporating both the potential partner connotation (although implicitly) and the attractiveness connotation. Our rationale for not making the potential partner connotation explicit in our phrasing was that including the word "partner" could be interpreted to equal a committed relationship, in contrast to e.g. a causal sexual relationship. Such a possible interpretation was not supported by the original phrasing and could therefore be a potential confounder in some cultural contexts. Hence, our version could be considered semantically more similar to the original version of SIAS item 14 than Weiss et al.'s. Whether this fine difference would yield any empirical difference in scores in any cultural context remains unexplored. Aside from validating a novel phrasing of item 14, the present study also extends previous research in several important aspects. First, the present study was performed on a clinical sample: considering that the SIAS is used in clinical contexts-as an outcome measure in clinical trials ( e.g. in [19]) and for screening purposes-a psychometric evaluation featuring a clinical sample was necessary before any revised edition was to be recommended for use in such populations. The present study also featured a more diverse set of other measures of well-being to ensure similar convergent validities of item versions. The rationale behind the rephrasing was also tested, albeit relying only on selfreported experience. Importantly, this study provides some initial cross-cultural validity to the rephrasing of heteronormative items in social anxiety self-rating scales.

Unfortunately, the SIAS is far from the only psychiatric instrument that features heteronormative language. In Sweden, the gold standard in assessing obsessivecompulsive disorder (OCD), the Yale-Brown Obsessive Compulsive Scale (Y-BOCS) [40], gave rise to a debate on heteronormativity and possible discrimination of sexual 
minorities in psychiatry [41]. The Y-BOCS features an item that screens for sexual obsessions with "content [that] involves homosexuality", the administration of which led to accusations of heteronormativity and pathologising homosexuality. Aside from the self-report measures of SAD noted by Weiss et al. [39], the widely used Symptom Checklist 90 [42] includes an item (number 21) similar in phrasing to SIAS item 14. Although the findings of the current study may be difficult to translate to the context of other psychiatric conditions (e.g. OCD), they do suggest that psychiatric rating scales can be revised with non-heteronormative formulations that do not confuse, insult or alienate sexual minority respondents.

This study has some limitations that should be recognised. First, the study design did not allow for investigation of test-retest reliability. While acknowledging that single-item analyses are particularly vulnerable to low test-retest reliability, other aspects of psychometric integrity in our study (e.g. internal consistencies, see Additional file 1: Table S2) were in line with previous studies that additionally showed sound test-retest reliability. Hence, we see no reason to suspect that the testretest reliability should be lower in the current study. Second, the sample size of 129 participants should be considered only moderate, although in line with previously published psychometric evaluations by our research group (e.g. [24]). Future studies with larger and more diverse samples would enable examining differential item functioning in different groups. In this study, participants were administered all rating scales via the Internet. However, since previous research has shown that the psychometric properties of the Internet-administered SIAS are similar to the pen-and-paper version [24], the results of this study should be equally valid for paperadministered versions of the SIAS. Finally, we cannot exclude the possibility of an impact of the cultural context in which this study was performed. While our non-heteronormative formulation of item 14 does not make any explicit reference to any sexual orientation, the phrasing does invite further interpretation and hence, cultural factors may be invoked. This study was conducted in Sweden, a country that relative to many other countries enjoys increasing acceptance and recognition of sexual minority individuals. Therefore the present study would benefit from replication in other languages and cultures.

\section{Conclusion}

This study concludes that the Social Interaction Anxiety Scale (SIAS) can be revised with the non-heteronormative formulation of item 14 herein described without affecting the psychometric properties. This non-heteronormative revision of SIAS is recommended for use in both clinical and research settings to avoid potentially alienating, confusing or insulting sexual minority respondents.

\section{Additional file}

Additional file 1: Table S1. Demographics and clinical characteristics of the sample and between-group statistical comparisons.Table S2. Internal consistencies (Cronbach's alphas) of all scales. Table S3. Matrix of correlation coefficients for each group seperately. Figure S1. Relative distributions of responses to the revised and original SIAS item 14 between those who answered "Yes" and "No" to the question "Do you find it more difficult talking to an attractive person if this person, on the basis of sex, is a potential partner?"

\section{Abbreviations}

ABM: Attention bias modification training; LSAS-SR: Liebowitz social anxiety scale self-rated; MADRS-S: Montgomery-Åsberg depression rating scale self-rated; SIAS: Social interaction anxiety scale; SPS: Social phobia scale; QOLI: Quality of life inventory.

\section{Competing interests}

The authors declare that they have no competing interests.

\section{Authors' contributions}

Authors PL and PC designed the study. PL carried out analyses and drafted the manuscript. JB carried out additional analyses. CM, JB, GA and PC made significant contributions to the study rationale and interpretation of findings. All authors participated in the review and revision of the manuscript and have approved the final manuscript to be published.

\section{Acknowledgements}

The authors thank all their sexual minority patients and study participants who over the years have voiced their reactions to the heteronormative SIAS. Thanks also to the participants and co-workers involved the SOFIE-13 intervention study which was made possible by a generous grant from the Swedish Council for Working Life and Social Research (FAS 2009-0222). An earlier version of this work was presented at a poster session at the Second European Meeting for the European Society for Research on Internet Interventions (ESRII) at Linköping University, Sweden, during October 2013.

\section{Author details}

${ }^{1}$ Department of Clinical Neuroscience, Karolinska Institutet, Karolinska, Sweden. ${ }^{2}$ Martell Behavioral Activation Research Consulting and Department of Psychology, University of Wisconsin, Milwaukee, WI, USA. ${ }^{3}$ Department of Psychology, Stockholm University, Stockholm, Sweden. ${ }^{4}$ Department of Behavioural Sciences and Learning, Psychology, Linköping University, Stockholm, Sweden.

Received: 27 May 2013 Accepted: 12 December 2013

Published: 19 December 2013

\section{References}

1. McIntyre M, McDonald C: The limitations of partial citizenship: health care institutions underpinned with heteronormative ideals. ANS Adv Nurs Sci 2012, 35:127-134.

2. Andersen R, Fetner $\mathrm{T}$ : Cohort differences in tolerance of homosexuality: attitudinal change in Canada and the United States, 1981-2000. Public Opin Q 2008, 72:311-330.

3. Röndahl G, Innala S, Carlsson M: Heterosexual assumptions in verbal and non-verbal communication in nursing. J Adv Nurs 2006, 56:373-381.

4. Bjorkman M, Malterud K: Lesbian women's experiences with health care: a qualitative study. Scand J Prim Health Care 2009, 27:238-243.

5. Chapman R, Wardrop J, Freeman P, Zappia T, Watkins R, Shields L: A descriptive study of the experiences of lesbian, gay and transgender parents accessing health services for their children. J Clin Nurs 2012, 21:1128-1135.

6. Bakker FC, Sandfort TGM, Vanwesenbeeck I, van Lindert H, Westert GP: Do homosexual persons use health care services more frequently than heterosexual persons: findings from a Dutch population survey. Soc Sci Med 2006, 63:2022-2030.

7. Pachankis JE, Bernstein LB: An etiological model of anxiety in young gay men: from early stress to public self-consciousness. Psychol Men Masc 2012, 13:107-122. 
8. Feinstein BA, Goldfried MR, Davila J: The relationship between experiences of discrimination and mental health among lesbians and gay men: an examination of internalized homonegativity and rejection sensitivity as potential mechanisms. J Consult Clin Psychol 2012, 80:917-927.

9. King M, McKeown E, Warner J, Ramsay A, Johnson K, Cort C, Wright L, Blizard R, Davidson O: Mental health and quality of life of gay men and lesbians in England and Wales: controlled, cross-sectional study. $\mathrm{Br}$ J Psychiatry 2003, 183:552-558.

10. Meyer $\mathrm{H}$ : Prejudice, social stress, and mental health in lesbian, gay, and bisexual populations: conceptual issues and research evidence. Psychol Bull 2003, 129:674-697.

11. Pachankis JE, Goldfried MR: Social anxiety in young gay men. J Anxiety Disord 2006, 20:996-1015.

12. Rapee RM, Spence SH: The etiology of social phobia: empirical evidence and an initial model. Clin Psychol Rev 2004, 24:737-767.

13. Mattick RP, Clarke JC: Development and validation of measures of social phobia scrutiny fear and social interaction anxiety. Behav Res Ther 1998, 36:455-470

14. DeBruine LM: Facial resemblance increases the attractiveness of samesex faces more than other-sex faces. Proc Biol Sci 2004, 271:2085-2090.

15. Fergus TA, Valentiner DP, McGrath PB, Gier Lonsway SL, Kim HS: Short forms of the Social Interaction Anxiety Scale and the Social Phobia Scale. J Pers Assess 2012, 94:310-320.

16. Kupper N, Denollet J: Social anxiety in the general population: introducing abbreviated versions of SIAS and SPS. J Affect Disord 2012 136:90-98.

17. Peters L, Sunderland M, Andrews G, Rapee RM, Mattick RP: Development of a short form Social Interaction Anxiety (SIAS) and Social Phobia Scale (SPS) using nonparametric item response theory: the SIAS-6 and the SPS-6. Psychol Assess 2012, 24:66-76.

18. Carleton RN, Collimore KC, Asmundson GJG, McCabe RE, Rowa K, Antony MM: Refining and validating the Social Interaction Anxiety Scale and the Social Phobia Scale. Depress Anxiety 2009, 26:E71-81.

19. Boettcher J, Andersson G, Carlbring P: Combining attention training with cognitive-behavior therapy in Internet-based self-help for social anxiety: study protocol for a randomized controlled trial. Trials 2013, 14:68.

20. Boettcher J, Hasselrot J, Sund E, Andersson G, Carlbring P, Boettcher J, Hasselrot J, Sund E, Andersson G, Carlbring P: Combining attention training with internet-based cognitive-behavioural self-help for social anxiety: a randomised controlled trial. Cogn Behav Ther 2013, 43:34-48

21. First MB, Spitzer RL, Miriam G, Williams JBW: Structured Clinical Interview for DSM-IV-TR Axis I Disorders, Research Version, Patient Edition (SCID-I/P). New York: Biometrics Research, New York State Psychiatric Institute; 2002.

22. Rohde P, Ph D, Lewinsohn PM, Seeley JR: Comparability of telephone and face-to-face interviews in assessing axis I and II disorders. Am J Psychiatry 1997, 154:1593-1598.

23. Carlbring P, Brunt S, Bohman S, Austin D, Richards J, Qst LG, Andersson G: Internet vs. paper and pencil administration of questionnaires commonly used in panic/agoraphobia research. Comput Human Behav 2007, 23:1421-1434.

24. Hedman E, Ljótsson B, Rück C, Furmark T, Carlbring P, Lindefors N, Andersson $\mathrm{G}$ : Internet administration of self-report measures commonly used in research on social anxiety disorder: a psychometric evaluation. Comput Human Behav 2010, 26:736-740.

25. Hirai M, Vernon LL, Clum GA, Skidmore ST: Psychometric properties and administration measurement invariance of social phobia symptom measures: paper-pencil vs. Internet administrations. J Psychopathol Behav Assess 2011, 33:470-479.

26. Lindner $P$, Andersson $G$, Ost L-G, Carlbring P: Validation of the InternetAdministered Quality of Life Inventory (QOLI) in different Psychiatric conditions. Cogn. Behav. Ther 2013, 42:315-327.

27. Thorndike FP, Carlbring P, Smyth FL, Magee JC, Gonder-Frederick L, Ost L-G, Ritterband LM: Web-based measurement: effect of completing single or multiple items per webpage. Comput Human Behav 2009, 25:393-401.

28. Peters L: Discriminant validity of the Social Phobia and Anxiety Inventory (SPAI), the Social Phobia Scale (SPS) and the Social Interaction Anxiety Scale (SIAS). Behav Res Ther 2000, 38:943-950.

29. Rodebaugh TL, Woods CM, Heimberg RG, Liebowitz MR, Schneier FR: The factor structure and screening utility of the Social Interaction Anxiety Scale. Psychol Assess 2006, 18:231-237.
30. Baker SL, Heinrichs N, Kim H-J, Hofmann SG: The liebowitz social anxiety scale as a self-report instrument: a preliminary psychometric analysis. Behav Res Ther 2002, 40:701-715.

31. Liebowitz MR: Social phobia. Mod Probl 1987, 22:141-173.

32. Svanborg P, Åsberg M: A new self-rating scale for depression and anxiety states based on the comprehensive Psychopathological rating scale. Acta Psychiatr Scand 1994, 89:21-28.

33. Frisch MB, Cornell J, Villanueva M, Retzlaff PJ: Clinical validation of the quality of life inventory. A measure of life satisfaction for use in treatment planning and outcome assessment. Psychol Assess 1992, 4:92-101.

34. Paunović N, Öst L-G: Clinical validation of the swedish version of the quality of life inventory in crime victims with posttraumatic stress disorder and a nonclinical sample. J Psychopathol Behav Assess 2004, 26:15-21.

35. Frisch MB, Clark MP, Rouse SV, Rudd MD, Paweleck JK, Greenstone A, Kopplin DA: Predictive and treatment validity of life satisfaction and the quality of life inventory. Assessment 2005, 12:66-78.

36. Carifio J, Perla RJ: Ten common misunderstandings, misconceptions, persistent myths and urban legends about likert scales and likert response formats and their antidotes. J Soc Sci 2007, 3:106-116.

37. Steiger $\mathrm{JH}$ : Tests for comparing elements of a correlation matrix. Psychol Bull 1980, 87:245-251.

38. Diedenhofen B: cocor: Comparing correlations; 2013

39. Weiss BJ, Hope DA, Capozzoli MC: Heterocentric language in commonly used measures of social anxiety: recommended alternate wording. Behav Ther 2013, 44:1-11.

40. Goodman WK, Price LH, Rasmussen SA, Mazure C, Fleischmann RL, Hill CL, Heninger GR, Charney DS: The yale-brown obsessive compulsive scale. I. Development, use, and reliability. Arch Gen Psychiatry 1989, 46:1006-1011.

41. Ruck C, Bergstrom J: Is the Y-BOCS discriminatory against gays and lesbians? Am J Psychiatry 2006, 163:1449.

42. Derogatis LR: Symptom Checklist-90-Revised, Pearson Clinical Assessment; 1994.

doi:10.1186/1477-7525-11-209

Cite this article as: Lindner et al.: Clinical validation of a

non-heteronormative version of the Social Interaction Anxiety Scale (SIAS). Health and Quality of Life Outcomes 2013 11:209.

\section{Submit your next manuscript to BioMed Central and take full advantage of:}

- Convenient online submission

- Thorough peer review

- No space constraints or color figure charges

- Immediate publication on acceptance

- Inclusion in PubMed, CAS, Scopus and Google Scholar

- Research which is freely available for redistribution 